\title{
Prevalence of RECQL germline variants in Pakistani early-onset and familial breast cancer patients
}

\author{
Muhammad Usman Rashid ${ }^{1,2^{*}}$, Noor Muhammad ${ }^{1}$, Faiz Ali Khan ${ }^{1}$, Umara Shehzad ${ }^{1}$, Humaira Naeemi ${ }^{1}$, \\ Naila Malkani ${ }^{3}$ and Ute Hamann ${ }^{2^{*}}$ (D)
}

\begin{abstract}
Background: The RecQ Like Helicase (RECQL) gene has previously been shown to predispose to breast cancer mainly in European populations, in particular to estrogen receptor (ER) and/or progesterone receptor (PR) positive tumor. Here, we investigated the contribution of pathogenic RECQL germline variants to hereditary breast cancer in early-onset and familial breast cancer patients from Pakistan.

Methods: Comprehensive RECQL variant analysis was performed in 302 BRCA1 and BRCA2 negative patients with ER and/or PR positive breast tumors using denaturing high-performance liquid chromatography followed by DNA sequencing. Novel variants were classified using Sherloc guidelines.

Results: One novel pathogenic protein-truncating variant (p.W75*) was identified in a 37-year-old familial breast cancer patient. The pathogenic variant frequencies were $0.3 \%(1 / 302)$ in early-onset and familial breast cancer patients and $0.8 \%(1 / 133)$ in familial patients. Further, three novel variants of unknown significance, p.I141F, p.S182S, and p.C475C, were identified in familial breast cancer patients at the age of 47, 68, and 47 respectively. All variants were absent in 250 controls.
\end{abstract}

Conclusions: Our data suggest that the RECQL gene plays a negligible role in breast cancer predisposition in Pakistan.

Keywords: RECQL, Germline variants, Breast cancer, Pakistan

\section{Background}

Globally, the incidence of breast cancer has increased to approximately 2 million cases in 2017, while the mortality rate between 2007 and 2017 has declined [1]. In Pakistan, breast cancer is the most frequent invasive malignancy among women, accounting for $36.8 \%$ of all female malignancies [2]. Pakistan has one of the highest

\footnotetext{
* Correspondence: usmanr@skm.org.pk; u.hamann@dkfz-heidelberg.de 'Department of Basic Sciences Research, Shaukat Khanum Memorial Cancer Hospital and Research Centre (SKMCH\&RC), 7A, Block R3, Johar Town, Lahore, Punjab 54000, Pakistan

${ }^{2}$ Molecular Genetics of Breast Cancer, German Cancer Research Center (DKFZ), Im Neuenheimer Feld 580, 69120 Heidelberg, Germany Full list of author information is available at the end of the article
}

rates of breast cancer in Asia, with age-standardized (world) annual incidence and mortality rates of 43.9 and 23.2 per 100,000, respectively [2]. Breast cancer incidence and mortality trends are still increasing [3, 4], making breast cancer a major public health burden in this developing country.

Approximately $50 \%$ of familial breast cancer is due to pathogenic germline variants in high, and moderate penetrance genes and common low-penetrance genetic variants [5]. Most of these genes are involved in the DNA repair pathway and maintenance of genomic stability, underlining the significance of other genes involved in this pathway. In 2015, the RecQ Like Helicase 
$(R E C Q L)$ gene was identified in West European and East Asian populations as a candidate breast cancer susceptibility gene [6, 7]. It encodes a DNA helicase, which is involved in the repair of DNA double-strand breaks and plays a crucial role in the maintenance of genomic stability. Several studies conducted among unselected breast cancer patients from Belarus and Germany [8], USA [9], and early-onset and familial breast cancer patients from Poland [6], Canada [6], and Australia [10] reported pathogenic $R E C Q L$ variant frequencies ranging from 0 to $2.6 \%$. Breast tumors associated with pathogenic $R E C Q L$ variants were predominantly positive for the estrogen and progesterone receptors (ER and PR) $[6-8,11]$.

Apart from two studies conducted in an East Asian population from China [7, 11], data on the contribution of pathogenic $R E C Q L$ variants to early-onset and/or familial breast cancer patients from other Asian regions are lacking. In Pakistan, breast cancer is the most common malignancy and main cause of cancer-related deaths in women. The burden of breast cancer in terms of estimated age-standardised incidence and mortality rates is 43.9 and 23.2 per 100,000 , respectively [12]. Pathogenic variants in high- and moderate-penetrance breast cancer susceptibility genes (BRCA1, BRCA2, TP53, CHEK2, RAD51C, and PALB2) account for about $27 \%$ of early-onset and familial breast cancers in Pakistan [13-17], leaving a substantial proportion of cases unexplained. In the present study, we determined the contribution of pathogenic $R E C Q L$ variants to hereditary breast cancer in 302 early-onset and familial BRCA1 and BRCA2 negative patients with ER positive and/or PR positive breast cancer in a South Asian population from Pakistan.

\section{Methods}

\section{Study subjects}

Patients diagnosed with invasive breast cancer were selected from the institutional registry of genetically enriched breast and ovarian cancer families enrolled at the Shaukat Khanum Memorial Cancer Hospital and Research Centre (SKMCH\&RC) in Lahore, Pakistan, from June 2001 to August 2015, fulfilling the inclusion criteria as described previously $[17,18]$. The present study included 302 early-onset and familial breast cancer patients with ER positive and/or PR positive tumors. All study participants were tested negative for pathogenic variants in $B R C A 1, B R C A 2[17,18]$ and about $60 \%$ for pathogenic variants in PALB2 $(n=187)$, TP53 $(n=180)$, CHEK2 $(n=168)$, and RAD51C $(\mathrm{n}=168) \quad$ [13-16] (Muhammad U. Rashid, unpublished TP53 data). We categorized study participants into four risk groups based on age at cancer diagnosis or family history of breast and/or ovarian cancer (Table 1) [17].
The control population comprised 250 healthy women with no family history of breast/ovarian cancer. They were selected from the institutional registry of 1012 female controls enrolled in a Pakistani breast cancer case-control study as previously described [19]. The Institutional Review Board (IRB) of the SKMCH\&RC approved the current study (IRB approval number ONCBRCA-001/2). All study participants signed informed written consent.

\section{Variant screening}

The complete coding sequence and exon-intron junctions of the RECQL gene (Genbank accession number NM_002907.3) were screened in the 302 index patients and 250 controls by denaturing high-performance liquid chromatography (DHPLC) analysis. The PCR primers details are described elsewhere [7]. When available, a positive control with a known variant was included in each set of DHPLC analysis. Bidirectional DNA sequencing was performed to confirm a variant, as described elsewhere [20].

\section{Variant classification}

The novel $R E C Q L$ variants were analyzed using the numerical score-based variant classification system Sherloc, a comprehensive refinement of the American College of Medical Genetics and Genomics-Association for Molecular Pathology (ACMG-AMP). Five evidence categories (two clinical and three functional) were used to evaluate variants. Clinical criteria include variant frequency information from large human population data, the Genome Aggregation Database (gnomAD; https://gnomad.broadinstitute.org/ gene/-ENSG00000004700?dataset=gnomad_r2_1) and variant observation in unaffected and affected individuals and families. For variants classification, allele frequencies of South Asian population from gnomAD were used as this population has ethnic and geographic relevance to Pakistani population. Functional criteria include variant type, experimental studies, and computational data. The following in silico tools for prediction of protein function or splicing were used: PolyPhen-2 (http://genetics.bwh.harvard.edu/ pph2/), SIFT (https://sift.bii.a-star.edu.sg/), SNAP2 (http:// www.rostlab.org/services/snap/submit), MutationTaster (http://www.mutationtaster.org/), SNPs\&GO (http://snps. biofold.org/snps-and-go/snps-and-go.html), and nsSNP Analyzer (http://snpanalyzer.uthsc.edu/) for the missense variants, $[14,16]$ and splice-site prediction algorithms MaxEntScan (http://genes.mit.edu/burgelab/maxent/Xmaxentscan_scoreseq.html), NNSPLICE (http://www.fruitfly.org/ seq_tools/splice.html), HumanSplice Finder (http://www. umd.be/HSF3/), GeneSplicer (http://ccb.jhu.edu/software/ genesplicer/), and SpliceSiteFinder-like (http://www.umd. be/searchSpliceSite.html) for splice-site and intronic variants [14]. In case of any disagreement between clinical and 
Table 1 Frequency of RECQL pathogenic variants according to family structure

\begin{tabular}{|c|c|c|c|}
\hline Risk group & Phenotype of families & $\begin{array}{l}\text { No. of } \\
\text { families }\end{array}$ & $\begin{array}{l}\text { Families with RECQL pathogenic variant } \mathrm{n} \\
\text { (\%) }\end{array}$ \\
\hline & All families & 302 & $1(0.3)$ \\
\hline$A 1+A 2+A 3$ & Female breast cancer families & 255 & $1(0.4)$ \\
\hline A1 & Early-onset breast cancer ( 1 case $\leq 30$ years) & 122 & $0(0.0)$ \\
\hline$A 2+A 3$ & Familial breast cancer ( $\geq 2$ cases, $\geq 1$ diagnosed $\leq 50$ years) & 133 & $1(0.8)$ \\
\hline A4 & Male breast cancer ( 1 case diagnosed at any age) & 29 & $0(0.0)$ \\
\hline B & $\begin{array}{l}\text { Breast and ovarian cancer families ( } \geq 1 \text { breast cancer and } \geq 1 \text { ovarian } \\
\text { cancer) }\end{array}$ & 18 & $0(0.0)$ \\
\hline
\end{tabular}

functional evidence, the clinical evidence was considered more convincing.

Variants were classified as pathogenic, likely pathogenic, benign, likely benign, and as variants of uncertain significance (VUS), according to the Sherloc guidelines [21]. Sherloc is a semiquantitative system in which each criterion is awarded a preset number of points on orthogonal benign (1B-5B) or pathogenic (1P-5P) scales using clinical and functional criteria. Point thresholds for pathogenic and benign classifications are $5 \mathrm{P}$ and $5 \mathrm{~B}$, for likely pathogenic and likely benign classifications $4 \mathrm{P}$ and $3 \mathrm{~B}$, and for VUS $<4 \mathrm{P}$ and $<3 \mathrm{~B}$. Pathogenicity and benign point scores were calculated separately.

\section{RNA analysis of the c.868-2A > G splice-site variant}

Total RNA was extracted from blood samples of the proband and an unaffected sister harboring the $R E C Q L$ c.868-2A > G, another variant negative unaffected sister, and a variant negative control using TRIzol reagent (Invitrogen, Carlsbad, CA, USA). Total RNA was transcribed to cDNA using the RevertAid First Strand cDNA Synthesis Kit (Thermo Fisher Scientific, Vilnius, Lithuania) with random hexamer primers according to the manufacturer's protocol. Reverse transcriptase (RT)PCR was performed using the forward primer $\left(5^{\prime}-\right.$ CAG TTC CCT AAC GCA TCA CT - 3') and reverse primer $\left(5^{\prime}\right.$ - TTT CAT TGG CTG ACC ATT TT - 3') located on exon 7 and exon 9 of the RECQL transcript variant 1 (ENST00000444129.7), respectively. PCR reactions were carried out in a $25 \mu \mathrm{l}$ volume containing $1 \mu \mathrm{l}$ of respective cDNA, 1x PCR Gold Buffer (Applied Biosystems, California, USA), $2.5 \mathrm{mM} \mathrm{MgCl}_{2}, 0.2 \mu \mathrm{M}$ of each primer, $250 \mu \mathrm{M}$ of each dNTP (Invitrogen, Carlsbad CA, USA), and 1 unit AmpliTaq Gold DNA polymerase (Applied Biosystems, California, USA). After an initial denaturation for $15 \mathrm{~min}$ at $95^{\circ} \mathrm{C}$, cDNA was amplified by 35 cycles of $1 \mathrm{~min}$ at $94^{\circ} \mathrm{C}, 1 \mathrm{~min}$ at $57.5^{\circ} \mathrm{C}, 1$ min at $72{ }^{\circ} \mathrm{C}$, and a final extension step of $5 \mathrm{~min}$ at $72{ }^{\circ} \mathrm{C}$. Five $\mu \mathrm{l}$ of RT-PCR products were loaded on a $2 \%$ agarose gel containing ethidium bromide (Sigma-
Aldrich, Steinheim, Germany) and electrophoresis was performed at $140 \mathrm{~V}$ for $80 \mathrm{~min}$ and confirmed by Sanger sequencing as described previously [20].

\section{Results}

\section{Characteristics of the study participants}

A total of 302 BRCA1 and BRCA2 negative index breast cancer patients were screened for $R E C Q L$ germline variants. Of these patients, $122(40.4 \%)$ were early-onset breast cancer patients ( $\leq 30$ years of age), $133(44.0 \%)$ belonged to families with two or more breast cancer cases with at least one case diagnosed at 50 years or younger, $18(6.0 \%)$ to families with both breast and ovarian cancer, and 29 (9.6\%) male breast cancer cases diagnosed at any age (Table 1). Of the index patients, 223 presented with ER positive and PR positive breast tumors, 55 with ER positive tumors, and 24 with PR positive tumors. The mean age of disease presentation was 36.6 years (range 20-78) for female breast cancer $(n=$ 273 ), and 51.5 years (range 27-73) for male breast cancer $(n=29)$.

\section{Spectrum of identified RECQL variants}

In total, 31 distinct $R E C Q L$ variants were detected. Of these, 20 were novel: one nonsense variant, one splicesite variant, three missense variants, three silent variants, and twelve noncoding variants (Table 2). The remaining eleven variants were previously reported: three missense variants and eight noncoding variants.

\section{Classification and characteristics of identified RECQL variants}

The novel variants were analyzed for their potential functional effect using Sherloc guidelines [21], including the minor allele frequency (MAF) $>1 \%$ for benign variants reported in Genome Aggregation Database (gnomAD) or in our study (Table 2) and in silico prediction tools (Table 3). One variant was classified as pathogenic, three as VUS, and 16 as benign/likely benign. 
Table 2 RECQL germline variants identified in the study cases and controls from Pakistan

\begin{tabular}{|c|c|c|c|c|c|c|c|c|c|}
\hline \multirow[t]{2}{*}{ Location } & \multirow{2}{*}{$\begin{array}{l}\text { Coding (c.) DNA } \\
\text { Sequence }^{\mathrm{a}}\end{array}$} & \multirow[t]{2}{*}{ Effect } & \multicolumn{2}{|c|}{ Prevalence $\mathbf{n}(\%)$} & \multicolumn{3}{|c|}{ MAF (\%) } & \multirow[t]{2}{*}{ Classification } & \multirow[t]{2}{*}{ Reference } \\
\hline & & & $\begin{array}{l}\text { Cases } \\
(n=302)\end{array}$ & $\begin{array}{l}\text { Controls } \\
(n=250)\end{array}$ & Cases & Controls & $\overline{\text { gnomAD, SAS }}$ & & \\
\hline \multicolumn{10}{|c|}{ Pathogenic variant } \\
\hline Exon 4 & c.225G > A (p.W75*) & Nonsense & $1(0.3)$ & $0(0.0)$ & 0.166 & 0 & NA & $p^{b}$ & Novel \\
\hline \multicolumn{10}{|c|}{ Variants of uncertain significance } \\
\hline Exon 5 & $c .421 \mathrm{~A}>\mathrm{T}(\mathrm{p} .1141 \mathrm{~F})$ & Missense & $1(0.3)$ & $0(0.0)$ & 0.166 & 0 & 0.0188 & Vus $s^{b}$ & Novel \\
\hline Exon 6 & c.546C > T (p.S182S) & Silent & $1(0.3)$ & $0(0.0)$ & 0.166 & 0 & 0.0165 & VUS $S^{b}$ & Novel \\
\hline Exon 12 & c. $1425 C>$ T (p.C475C) & Silent & $1(0.3)$ & $0(0.0)$ & 0.166 & 0 & 0.0033 & VUS $s^{b}$ & Novel \\
\hline \multicolumn{10}{|l|}{ Benign variants } \\
\hline \multicolumn{10}{|l|}{ Coding } \\
\hline Exon 3 & c.132G > A (p.K44K) & Silent & $34(11.3)$ & $28(11.2)$ & 5.629 & 5.6 & NA & B & Novel \\
\hline Exon 3 & c.151G > A (p.E51K) & Missense & $49(16.2)$ & $34(13.6)$ & 8.113 & 6.8 & 0.0098 & B & Novel \\
\hline Exon 7 & c.833C > G (p.T278R) & Missense & $5(1.7)$ & $6(2.4)$ & 0.828 & 1.2 & 1.6417 & B & ClinVar, [10] \\
\hline Exon 8 & c.898 T > A (p.F300I) & Missense & $1(0.3)$ & $0(0.0)$ & 0.166 & 0 & 0.0033 & B & {$[6,10]$} \\
\hline Exon 13 & c.1651A > G (p.1551V) & Missense & $1(0.3)$ & $1(0.4)$ & 0.166 & 0.2 & 0.0785 & $L B^{b}$ & Novel \\
\hline Exon 13 & c.1661A > G (p.Y554C) & Missense & $5(1.7)$ & $1(0.4)$ & 0.828 & 0.2 & 0.2366 & LB & [10] \\
\hline \multicolumn{10}{|c|}{ Non-coding variants } \\
\hline $5^{\prime} \mathrm{UTR}$ & $c .-110 G>A$ & $5^{\prime}$ UTR & $2(0.7)$ & $3(1.2)$ & 0.331 & 0.6 & NA & B & Novel \\
\hline $5^{\prime} \mathrm{UTR}$ & c. $-137 \mathrm{C}>\mathrm{T}$ & $5^{\prime} \mathrm{UTR}$ & $3(1.0)$ & $3(1.2)$ & 0.497 & 0.6 & NA & B & ClinVar, [22] \\
\hline $5^{\prime} \mathrm{UTR}$ & c. $-187 \mathrm{~T}>\mathrm{G}$ & $5^{\prime}$ UTR & $1(0.3)$ & $0(0.0)$ & 0.166 & 0 & NA & B & Novel \\
\hline Intron 3 & c. $215-169 C>A$ & Intronic & $22(7.3)$ & $20(8.0)$ & 3.642 & 4.0 & 0 & LB & ClinVar \\
\hline Intron 3 & c. $215-86 G>A$ & Intronic & $1(0.3)$ & $1(0.4)$ & 0.166 & 0.2 & 0 & B & [22] \\
\hline Intron 3 & c. $215-48 C>A$ & Intronic & $1(0.3)$ & $1(0.4)$ & 0.166 & 0.2 & 0.0663 & B & Novel \\
\hline Intron 3 & c. $215-37 \mathrm{~T}>\mathrm{C}$ & Intronic & $1(0.3)$ & $0(0.0)$ & 0.166 & 0 & 0.0174 & B & Novel \\
\hline Intron 6 & c. $700+110 C>G$ & Intronic & $1(0.3)$ & $0(0.0)$ & 0.166 & 0 & NA & B & Novel \\
\hline Intron 7 & c.868-11G > A & Intronic & $1(0.3)$ & $1(0.4)$ & 0.166 & 0.2 & 0.0611 & B & Novel \\
\hline Intron 7 & c. $868-2 A>G$ & Intronic & $3(1.0)$ & $2(0.8)$ & 0.497 & 0.4 & 0.5669 & $\mathrm{~B}^{\mathrm{b}}$ & Novel \\
\hline Intron 8 & $c .949+62 A>G$ & Intronic & $1(0.3)$ & $0(0.0)$ & 0.166 & 0 & NA & B & Novel \\
\hline Intron 8 & $c .949+76 A>G$ & Intronic & $6(2.0)$ & $6(2.4)$ & 0.993 & 1.2 & 0 & LB & ClinVar \\
\hline Intron 11 & C. $1355+30 \mathrm{~T}>\mathrm{C}$ & Intronic & $13(4.3)$ & $17(6.8)$ & 2.152 & 3.4 & 51.4 & B & [22] \\
\hline Intron 11 & $c .1355+103 G>C$ & Intronic & $5(1.7)$ & $11(4.4)$ & 0.828 & 2.2 & 0 & B & ClinVar, [22] \\
\hline Intron 12 & c. $1448-18 A>G$ & Intronic & $1(0.3)$ & $0(0.0)$ & 0.166 & 0 & 0 & B & Novel \\
\hline Intron 13 & c. $1667+53 \mathrm{~T}>\mathrm{A}$ & Intronic & $2(0.7)$ & $1(0.4)$ & 0.331 & 0.2 & 0 & B & ClinVar \\
\hline Intron 13 & c. $1667+53$ delT & Intronic & $1(0.3)$ & $0(0.0)$ & 0.166 & 0 & 0 & B & Novel \\
\hline Intron 13 & c. $1668-160 C>T$ & Intronic & $1(0.3)$ & $0(0.0)$ & 0.166 & 0 & 0 & B & Novel \\
\hline Intron 13 & c. $1668-81 G>A$ & Intronic & $3(1.0)$ & $4(1.6)$ & 0.497 & 0.8 & 0 & B & Novel \\
\hline Intron 14 & c.1797+14_17delAATT & Intronic & $20(6.6)$ & $22(8.8)$ & 3.311 & 4.4 & 4.0024 & B & Novel \\
\hline 3'UTR & $c .{ }^{*} 6 \mathrm{~A}>\mathrm{C}$ & 3'UTR & $61(20.2)$ & $84(33.6)$ & 10.099 & 16.8 & NA & B & [22] \\
\hline
\end{tabular}

$B$ Benign, gnom $A D$ Genome aggregation database, $L B$ Likely benign, $L P$ Likely pathogenic, MAF Minor allele frequency, NA Not available, $P$ Pathogenic, SAS South Asians, VUS Variant of uncertain significance

${ }^{a}$ Nomenclature follows Human Genome Variation Society (HGVS) (http://www.hgvs.org). Numbering start at the first A of the first coding ATG (located in exon 2) of NCBI GenBank Accession NM 002907.3

${ }^{\mathrm{b}}$ Classification of nucleotide alterations was performed using Sherloc guidelines [21]

\section{Pathogenic RECQL variant}

The novel pathogenic $R E C Q L$ variant is a nonsense variant at nucleotide position 225 in exon 4 (c.225G > A $\left.\left(\mathrm{p} . \mathrm{W} 75^{*}\right)\right)$, which is predicted to result in premature protein termination. It was identified in a 37-year-old familial breast cancer patient (III:3, Fig. 1a) of Punjabi 
Table 3 In silico analyses of novel RECQL variants identified in the study cases from Pakistan

\begin{tabular}{|c|c|c|c|c|c|c|c|}
\hline \multirow[t]{2}{*}{ Coding variants } & \multicolumn{6}{|c|}{ In silico prediction } & \multirow[t]{2}{*}{ Consensus $^{a}$} \\
\hline & PolyPhen-2 & SIFT & SNAP2 & MutationTaster & SNPs\&GO & nsSNP Analyzer & \\
\hline c.151G > A (p.E51K) & Benign & Tolerated & Neutral & Disease causing & Neutral & Neutral & Benign \\
\hline$c .421 \mathrm{~A}>\mathrm{T}(\mathrm{p} .1141 \mathrm{~F})$ & Probably damaging & Deleterious & Effect & Disease causing & Disease & Disease & Deleterious (6/6) \\
\hline c.1651A > G (p.1551V) & Benign & Tolerated & Neutral & Polymorphism & Neutral & Neutral & Benign \\
\hline \multirow[t]{2}{*}{ Noncoding variants } & \multicolumn{6}{|c|}{ Splice-site predictions } & Consensus ${ }^{\mathrm{a}, \mathrm{b}}$ \\
\hline & SpliceSiteFinder-like & MaxEntScan & NNSPLICE & GeneSplicer & \multicolumn{2}{|c|}{ HumanSplice Finder } & \\
\hline c. $-110 G>A$ & $\mathrm{NE}$ & $\mathrm{NE}$ & $\mathrm{NE}$ & $\mathrm{NE}$ & \multicolumn{2}{|l|}{$\mathrm{NE}$} & Benign \\
\hline c. $-187 \mathrm{~T}>\mathrm{G}$ & $\mathrm{D}(0 \rightarrow 73.0)$ & $\mathrm{D}(0 \rightarrow 2.9)$ & $\mathrm{NE}$ & $\mathrm{NE}$ & \multicolumn{2}{|l|}{$\mathrm{NE}$} & Benign \\
\hline c. $215-48 C>A$ & $\mathrm{NE}$ & $\mathrm{NE}$ & NE & $\mathrm{NE}$ & \multicolumn{2}{|l|}{$\mathrm{NE}$} & Benign \\
\hline c.215-37 T >C & $\mathrm{NE}$ & $\mathrm{NE}$ & $\mathrm{NE}$ & $\mathrm{NE}$ & \multicolumn{2}{|l|}{$\mathrm{NE}$} & Benign \\
\hline$c .700+110 C>G$ & $\mathrm{NE}$ & $\mathrm{NE}$ & NE & $\mathrm{NE}$ & \multicolumn{2}{|l|}{$\mathrm{A}(0 \rightarrow 80.1)$} & Benign \\
\hline c.868-11G > A & $\mathrm{A}(0 \rightarrow 85.8)$ & $\mathrm{A}(2.5 \rightarrow 7.1)$ & $\mathrm{NE}$ & $\mathrm{NE}$ & \multicolumn{2}{|l|}{$\mathrm{NE}$} & Benign \\
\hline$c .868-2 A>G$ & $\mathrm{~A}(0 \rightarrow 79.9)^{\mathrm{c}}$ & $\mathrm{A}(0 \rightarrow 5.4)^{c}$ & $\mathrm{~A}(0 \rightarrow 0.4)^{\mathrm{C}}$ & $\mathrm{NE}$ & \multicolumn{2}{|l|}{ NE } & Deleterious (3/5) \\
\hline$c .949+62 A>G$ & NE & NE & NE & $\mathrm{NE}$ & \multicolumn{2}{|l|}{$\mathrm{NE}$} & Benign \\
\hline c. $1448-18 A>G$ & $\mathrm{NE}$ & $\mathrm{NE}$ & $\mathrm{NE}$ & $\mathrm{NE}$ & \multicolumn{2}{|l|}{$\mathrm{NE}$} & Benign \\
\hline c. $1667+53$ delT & $\mathrm{NE}$ & $\mathrm{NE}$ & $\mathrm{NE}$ & NE & \multicolumn{2}{|l|}{ NE } & Benign \\
\hline c. $1668-160 C>T$ & NE & $\mathrm{D}(2.9 \rightarrow 1.2)$ & NE & NE & \multicolumn{2}{|l|}{$\mathrm{NE}$} & Benign \\
\hline c. $1668-81 G>A$ & $\mathrm{NE}$ & $\mathrm{NE}$ & $\mathrm{NE}$ & $\mathrm{NE}$ & \multicolumn{2}{|l|}{$\mathrm{NE}$} & Benign \\
\hline c.1797 + 14_17delAATT & $\mathrm{NE}$ & $\mathrm{A}(4.8 \rightarrow 2.3)$ & $\mathrm{NE}$ & $\mathrm{NE}$ & \multicolumn{2}{|l|}{$\mathrm{NE}$} & Benign \\
\hline
\end{tabular}

A Acceptor, $D$ Donor, NE No effect

${ }^{a}$ The variant is considered as deleterious by six of the six protein function prediction or three of the five splice-site prediction algorithms for coding or noncoding variants, respectively

${ }^{b}>20 \%$ change in score (i.e., a wild-type splice-site score decreases and/or a cryptic splice-site score increases) is considered significant

${ }^{c}$ Canonical splice acceptor site is abolished (MaxEntScan score $+2.46 \rightarrow-5.49$ ) and creates a cryptic splice acceptor site at c.877

ethnicity and was absent in 250 controls. The patient carrying this variant presented with a grade 3 , ER positive and PR positive invasive ductal carcinoma (IDC) with lymph node involvement. The pathogenic variant frequencies were $0.3 \%(1 / 302)$ in early-onset and familial breast cancer patients and $0.8 \%(1 / 133)$ in familial patients. The variant had a Sherloc score of $8 \mathrm{P}$ and was classified as pathogenic (Table 4).

\section{RECQL variants of uncertain significance (VUS)}

One novel missense variant (p.I141F) was identified in a 47-year-old familial breast cancer patient (II:4, Fig. 1b) of Punjabi origin. Two silent variants (p.S182S and p.C475C) were detected in familial breast cancer patients at age 68 (I:1, Fig. 1c) and 47 (III:10, Fig. 1d) respectively of Saraiki background. These variants were not detected in 250 controls. The population allele frequencies of p.I141F, p.S182S, and p.C475C were low $(\mathrm{MAF}=0.0188 \%, \mathrm{MAF}=$ $0.0165 \%$ and $\mathrm{MAF}=0.0033 \%$, respectively) and within the pathogenic range of $<8$ total alleles among South Asians $(n \geq 12,086)$ in the gnomAD. The missense variant had a Sherloc score of 2.5P and both silent variants of P1.5 and B3. All variants were classified as VUS (Table 4).

\section{Benign or likely benign variants}

One novel variant in a canonical splice acceptor site of intron 7, c.868-2A > G, was detected in a 36-year-old familial (II:4, Fig. 1e), a 61-year-old male (II:8, Fig. 1f), and a 25-year-old female early-onset breast cancer patient (II:9, Fig. 1g) of Punjabi, Urdu speaking and Pathan ethnicity, respectively $(1 \%, 3 / 302)$. It was also found in one of the two tested unaffected sisters (II:7, Fig. 1g) of the early-onset patient. Moreover, c.868-2A $>\mathrm{G}$ was detected in two controls $(0.8 \%, 2 / 250)$. The similar frequencies in cases and controls indicate that this variant is not likely to be pathogenic. Using the Sherloc guidelines, a high frequency of the $\mathrm{G}$ allele $(\mathrm{MAF}=0.5669 \%)$ was reported among South Asians $(n=13,582)$ in the gnomAD. It was predicted to have a functional impact by three of five splice-site prediction tools (Table 3).

To address if c.868-2A $>$ G affects splicing, RT-PCR analysis of RNA extracted from two variant carriers and two non-carriers (one family member and one control) revealed the presence of one transcript corresponding to the reference full-length transcript ( $364 \mathrm{bp}$ ) in all samples (Fig. 2a). All transcripts were confirmed by Sanger sequencing (Fig. 2b-e). Thus, this variant may not affect 


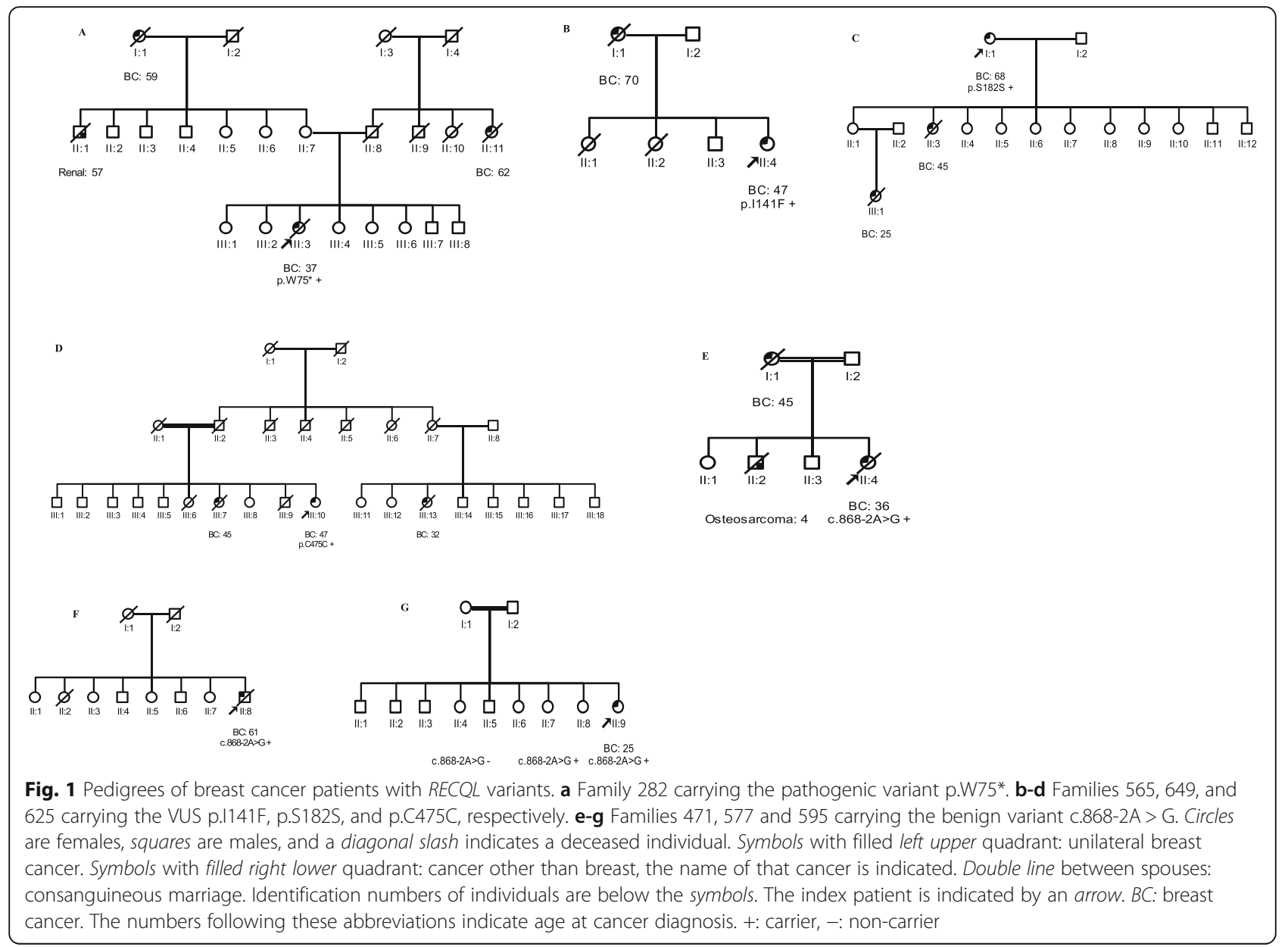

the splicing of RECQL. It had a Sherloc score of $1 \mathrm{P}$ and $8 \mathrm{~B}$ and was classified as benign (Table 4).

The remaining eleven variants (three missense variants and eight noncoding variants) have been previously reported as benign/likely benign in the ClinVar database (by April 2020) or in other populations.

\section{Discussion}

This is the first study that investigates the prevalence of pathogenic RECQL germline variants in $302 B R C A 1$ and $B R C A 2$ negative high-risk patients with ER positive and/ or PR positive breast tumors from Pakistan. We identified a single novel pathogenic RECQL variant. Although several studies had been previously conducted in Europe and only two studies in East Asia, there is still conflicting evidence for a role of $R E C Q L$ in breast cancer predisposition $[6,8,10,23]$. Our study provides additional information on the contribution of the RECQL gene to hereditary breast cancer in a South Asian population from Pakistan.

The novel pathogenic RECQL variant, p.W75* was identified in $0.3 \%$ of early-onset and familial breast cancer patients with hormone receptor-positive tumors, but not in controls, suggesting that p.W75* may be diseasecausative. In other studies performed in China [7, 11], higher pathogenic variant frequencies ranging from 0.54 to $1.6 \%$ were observed in BRCA1 and BRCA2 negative early-onset and/or familial breast cancer cases. In Caucasian studies conducted in the Australia [10], Canada [6], Poland [6], and USA [9], similar variant frequencies ranging from 0.1 to $0.4 \%$ have been reported in familial breast cancer patients, while no pathogenic variants were detected in studies performed in South-West Poland and West Ukraine [24]. In other Caucasian studies conducted in Belarus, Germany, and Australia, the frequency of pathogenic variants identified in controls were similar or higher than cases $[8,10]$. Overall, these findings suggest a controversial role of $R E C Q L$ as a breast cancer susceptibility gene.

Previously, a missense variant (p.R215Q) in the highly conserved RecA-like domain D1 of RECQL (amino acid residues 63 to 281) is reported to disrupt the RECQL helicase activity and classified as a pathogenic mutation [7]. In the current study, a novel missense variant, p.I141F, in the same domain was found in one familial breast cancer patient $(0.3 \%)$, but not in controls. It may 
Table 4 Sherloc variant classification criteria of novel RECQL variants

\begin{tabular}{|c|c|c|c|c|c|}
\hline Variant & Evidence \# & $\mathrm{P} / \mathrm{B}$ & Points score ${ }^{a}$ & Evidence type & Category \\
\hline \multirow[t]{6}{*}{ c.225G > A (p.W75*) } & EV0135 & $P$ & 1 & Clinical & Population - Frequency \\
\hline & EV0211 & $P$ & 0 & Clinical & Observation in Affecteds \\
\hline & EV0206 & $P$ & 2 & Clinical & Observation in Affecteds \\
\hline & EV0016 & $P$ & 5 & Functional & Variant Effect \\
\hline & Sum & & $8 \mathrm{P}$ & & \\
\hline & Sherloc score & & Pathogenic & & \\
\hline \multirow[t]{6}{*}{ c. $421 \mathrm{~A}>\mathrm{T}(\mathrm{p} .1141 \mathrm{~F})$} & EV0101 & $P$ & 0.5 & Clinical & Population - Frequency \\
\hline & EV0211 & $P$ & 0 & Clinical & Observation in Affecteds \\
\hline & EV0172 & $P$ & 1 & Functional & Variant Effect \\
\hline & EV0121 & $P$ & 1 & Functional & Computational \& Predictive \\
\hline & Sum & & $2.5 \mathrm{P}$ & & \\
\hline & Sherloc score & & VUS & & \\
\hline \multirow[t]{7}{*}{ c.546C > T (p.S182S) } & EV0101 & $P$ & 0.5 & Clinical & Population - Frequency \\
\hline & EV0211 & $P$ & 0 & Clinical & Observation in Affecteds \\
\hline & EV0193 & $P$ & 1 & Clinical & Observation in Affecteds \\
\hline & EV0103 & B & 2 & Functional & Variant Effect \\
\hline & EV0191 & B & 1 & Functional & Computational \& Predictive \\
\hline & Sum & & $1.5 \mathrm{P}, 3 \mathrm{~B}$ & & \\
\hline & Sherloc score & & VUS & & \\
\hline \multirow[t]{7}{*}{ c. 1425 C > T (p.C475C) } & EV0101 & $P$ & 0.5 & Clinical & Population - Frequency \\
\hline & EV0211 & $P$ & 0 & Clinical & Observation in Affecteds \\
\hline & EV0193 & P & 1 & Clinical & Observation in Affecteds \\
\hline & EV0103 & B & 2 & Functional & Variant Effect \\
\hline & EV0191 & B & 1 & Functional & Computational \& Predictive \\
\hline & Sum & & $1.5 \mathrm{P}, 3 \mathrm{~B}$ & & \\
\hline & Sherloc score & & VUS & & \\
\hline \multirow[t]{6}{*}{ c. $868-2 A>G$} & EV0096 & B & 5 & Clinical & Population - Frequency \\
\hline & EV0053 & B & 2 & Clinical & Observation in Unaffected \\
\hline & EV0037 & B & 1 & Functional & Functional Experiment \\
\hline & EV0187 & $P$ & 1 & Functional & Computational \& Predictive \\
\hline & Sum & & $1 \mathrm{P}, 8 \mathrm{~B}$ & & \\
\hline & Sherloc score & & Benign & & \\
\hline
\end{tabular}

$B$ Benign, EV Evidence, $P$ Pathogenic, VUS Variant of unknown significance

Pathogenicity and benign point scores are calculated separately

${ }^{\mathrm{a}}$ The Sherloc point score thresholds for pathogenic and benign classifications are $5 \mathrm{P}$ and $5 \mathrm{~B}$, and for VUS $<4 \mathrm{P}$ and $<3 \mathrm{~B}$

also affect the ATP-dependent translocation activity of RECQL leading to disruption of helicase activity [25]. However, functional assays are warranted to confirm this finding. Nevertheless, the population allele frequency of p.I141F was rare among South Asians in the gnomAD. Overall population data, variant type, clinical observation and findings from in silico predictions suggest that p.I141F may be a VUS based on the Sherloc guidelines.

The recurrent splice-site variant, c.868-2A > G, was identified in three breast cancer patients (1.0\%) and two controls $(0.8 \%)$. Its similar frequency in cases and controls indicates that this variant may be benign. This is supported by the fact that it has a very high frequency $(0.5669 \%)$ among South Asians in the gnomAD. In addition, RT-PCR analysis revealed that it did not affect the RECQL splicing. Thus, based on the Sherloc variant classification guidelines, our data suggest that c.868-2A $>\mathrm{G}$ may be benign. However, we cannot exclude that the aberrantly spliced allele may have escaped from detection due to the nonsense-mediated decay or other splicing events may 


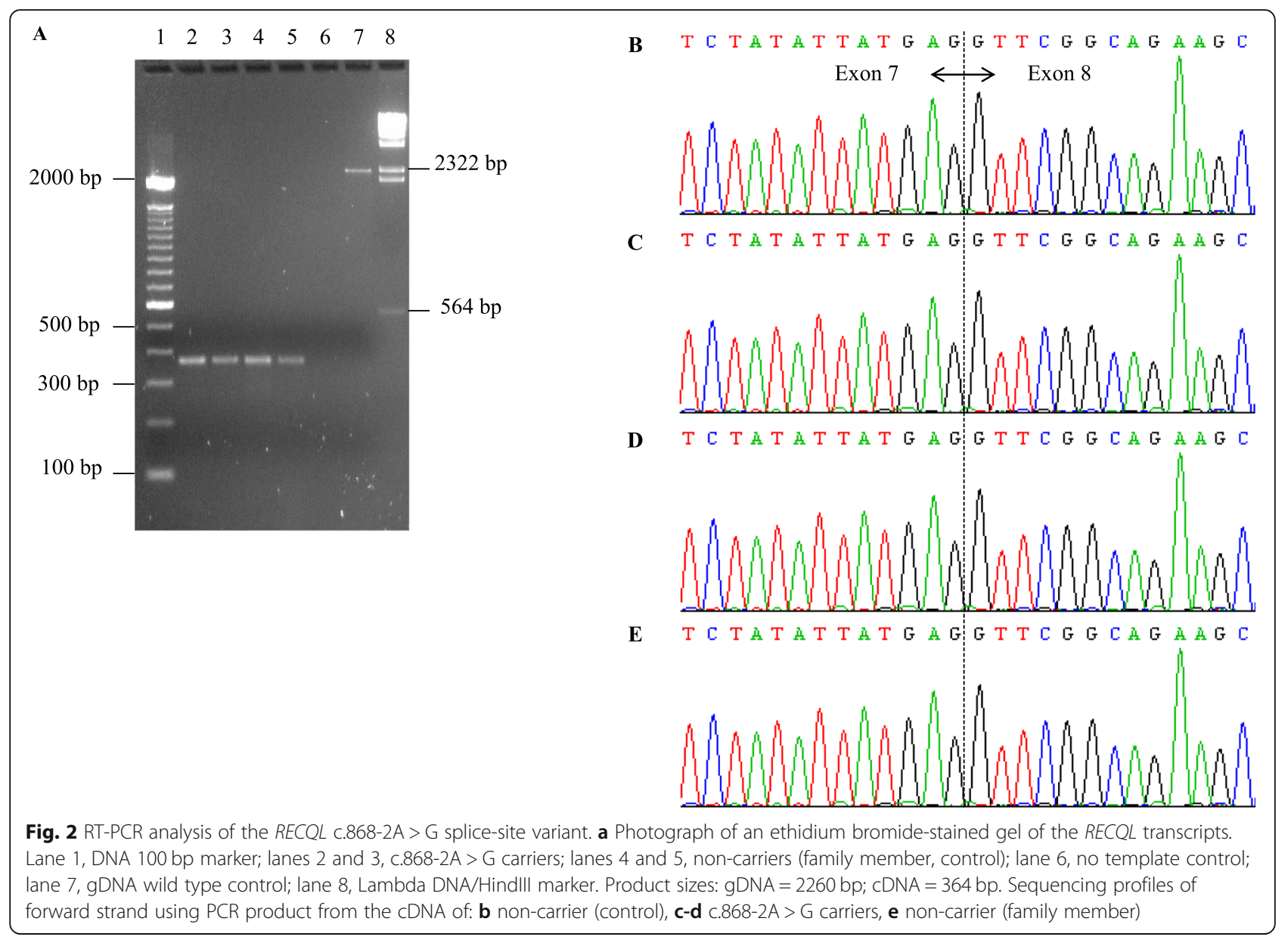

have occurred that were not investigated in the present study.

The ER and PR positive breast tumor of the Pakistani patient with the pathogenic RECQL variant showed high grade and IDC histology. These findings are in line with those from other studies conducted in China [7], Poland [6], Belarus, and Germany [8] further supporting the notion that high grade, hormone receptor-positive breast tumors of IDC histology may be predictors of the pathogenic $R E C Q L$ variant status.

Our study has several limitations. First, despite its reasonable size, larger studies are warranted to confirm our findings. Second, mutation analysis was restricted to patients with ER and/or PR positive breast tumors, in whom a predominance of pathogenic RECQL mutations has been reported [6-8, 11]. However, since patients with both ER and PR negative or triple-negative breast tumors were not tested, this may have undermined the prevalence of pathogenic RECQL variants reported in this study. Further, the functional analyses of the splice-site variant should be extended in order to confirm its classification as benign.

\section{Conclusion}

In summary, we identified a single pathogenic RECQL variant in $302 B R C A 1$ and $B R C A 2$ negative high-risk patients with ER positive and/or PR positive breast tumors. The frequencies of the novel pathogenic variant were $0.3 \%(1 / 302)$ in early-onset and familial breast cancer patients and $0.8 \%(1 / 133)$ in familial patients. Our data suggest that pathogenic RECQL variants explain a negligible proportion of hereditary breast cancer in Pakistan.

\section{Abbreviations}

ACMG-AMP: American College of Medical Genetics and GenomicsAssociation for Molecular Pathology; DHPLC: Denaturing high-performance liquid chromatography; ER: Estrogen receptor; gnomAD: Genome

Aggregation Database; IDC: Invasive ductal carcinoma; IRB: Institutional Review Board; MAF: Minor allele frequency; PR: Progesterone receptor; RECQ L: RecQ Like Helicase; RT-PCR: Reverse transcriptase polymerase chain reaction; SKMCH\&RC: Shaukat Khanum Memorial Cancer Hospital and Research Centre; VUS: Variants of uncertain significance

\section{Acknowledgements}

We are grateful to all study subjects for their participation. We thank the clinicians (Neelam Siddiqui, Mazhar Ali Shah, Narjis Muzaffar, Usman Ahmad, Umm E Kalsoom, Amir Ali Syed, Huma Majeed, Zulqarnain Chaudhry, Muhammad Asad Parvaiz, and Amina Khan) and clinical research officers (Saima Faisal and Mariam Hassan) for their help in recruitment of study 
participants. We thank Anna Jakubowska and Mohammad R. Akbari for providing DNA samples of RECQL variant carriers as positive controls for DHPLC analysis. We thank Jörg Hoheisel for critical reading of the manuscript. This study was funded by Shaukat Khanum Memorial Cancer Hospital and Research Centre, Lahore, Pakistan (grant number ONC-BRCA001/2) and the German Cancer Research Center, Heidelberg, Germany.

\section{Authors' contributions}

The study was designed by UH and MUR. Material preparation, data acquisition, and molecular analyses were performed by NM, US, FAK, and HN. mRNA analysis was done by NMa. Data was analyzed and interpreted by $\mathrm{UH}$, MUR, and NM. Manuscript was written by NM, MUR, and UH. Manuscript was reviewed and approved by all authors.

\section{Funding}

The study was funded by the Shaukat Khanum Memorial Cancer Hospital and Research Centre (grant number ONC-BRCA-001/002) and supported by the German Cancer Research Center. Open Access funding enabled and organized by Projekt DEAL.

\section{Availability of data and materials}

The data stated in this article including clinical and phenotypic characteristics of breast cancer families are confidential. Data are available at the Laboratory of Basic Sciences Research supervised by Dr. Muhammad Usman Rashid (usmanr@skm.org.pk), for researchers who meet the criteria to access confidential data. All information that support our findings are included within this article.

\section{Ethics approval and consent to participate}

All procedures performed in studies involving human participants were in accordance with the ethical standards of the institutional and/or national research committee and with the 1964 Helsinki declaration and its later amendments or comparable ethical standards. Written informed consent was obtained from all individual participants included in the study.

\section{Consent for publication}

Not applicable.

\section{Competing interests}

The authors declare that they have no competing interests.

\section{Author details}

'Department of Basic Sciences Research, Shaukat Khanum Memorial Cancer Hospital and Research Centre (SKMCH\&RC), 7A, Block R3, Johar Town, Lahore, Punjab 54000, Pakistan. ${ }^{2}$ Molecular Genetics of Breast Cancer, German Cancer Research Center (DKFZ), Im Neuenheimer Feld 580, 69120 Heidelberg, Germany. ${ }^{3}$ Department of Zoology, Government College University, Lahore, Pakistan

\section{Received: 14 September 2020 Accepted: 11 December 2020}

Published online: 20 December 2020

\section{References}

1. Li N, Deng Y, Zhou L, Tian T, Yang S, Wu Y, et al. Global burden of breast cancer and attributable risk factors in 195 countries and territories, from 1990 to 2017: results from the global burden of disease study 2017. J Hematol Oncol. 2019:12(1):140.

2. GLOBOCAN 2018: (Accessed 12 Nov 2020). Available from: https://gco.iarc.fr/ today/data/factsheets/populations/586-pakistan-fact-sheets.pdf.

3. Mubarik S, Malik SS, Wang Z, Li C, Fawad M, Yu C. Recent insights into breast cancer incidence trends among four Asian countries using ageperiod-cohort model. Cancer Manag Res. 2019;11:8145-55.

4. Mubarik S, Wang F, Fawad M, Wang Y, Ahmad I, Yu C. Trends and projections in breast cancer mortality among four Asian countries (1990-2017): evidence from five stochastic mortality models. Sci Rep. 2020;10(1):1-12

5. Couch FJ, Nathanson KL, Offit K. Two decades after BRCA: setting paradigms in personalized cancer care and prevention. Science. 2014; 343(6178):1466-70.
6. Cybulski C, Carrot-Zhang J, Kluzniak W, Rivera B, Kashyap A, Wokolorczyk D, et al. Germline RECQL mutations are associated with breast cancer susceptibility. Nat Genet. 2015;47(6):643-6.

7. Sun J, Wang Y, Xia Y, Xu Y, Ouyang T, Li J, et al. Mutations in RECQL gene are associated with predisposition to breast Cancer. PLoS Genet. 2015;11(5): e1005228.

8. Bogdanova N, Pfeifer K, Schurmann P, Antonenkova N, Siggelkow W, Christiansen $\mathrm{H}$, et al. Analysis of a RECQL splicing mutation, C.1667_1667+ 3delAGTA, in breast cancer patients and controls from Central Europe. Familial Cancer. 2017;16(2):181-6.

9. Chandler MR, Bergstresser S, Huskey ALW, Stallworth E, Davis A, Dean H, et al. Abstract A29: investigation of RECQL variants in European and African American breast cancer cohorts. Mol Cancer Res. 2018;16(8 Supplement): A29.

10. Li N, Rowley SM, Goode DL, Amarasinghe KC, Mclnerny S, Devereux L, et al. Mutations in RECQL are not associated with breast cancer risk in an Australian population. Nat Genet. 2018;50(10):1346-8.

11. Kwong A, Shin VY, Cheuk IWY, Chen J, Au CH, Ho DN, et al. Germline RECQL mutations in high risk Chinese breast cancer patients. Breast Cancer Res Treat. 2016;157(2):211-5.

12. Bray F, Ferlay J, Soerjomataram I, Siegel RL, Torre LA, Jemal A. Global cancer statistics 2018: GLOBOCAN estimates of incidence and mortality worldwide for 36 cancers in 185 countries. CA Cancer J Clin. 2018;68(6):394-424.

13. Rashid MU, Gull S, Asghar K, Muhammad N, Amin A, Hamann U. Prevalence of TP53 germ line mutations in young Pakistani breast cancer patients. Familial Cancer. 2012:11(2):307-11.

14. Rashid MU, Khan FA, Muhammad N, Loya A, Hamann U. Prevalence of PALB2 Germline mutations in early-onset and familial breast/ovarian Cancer patients from Pakistan. Cancer Res Treat. 2019;51(3):992-1000.

15. Rashid MU, Muhammad N, Faisal S, Amin A, Hamann U. Constitutional CHEK2 mutations are infrequent in early-onset and familial breast/ovarian cancer patients from Pakistan. BMC Cancer. 2013;13:312.

16. Rashid MU, Muhammad N, Faisal S, Amin A, Hamann U. Deleterious RAD51C germline mutations rarely predispose to breast and ovarian cancer in Pakistan. Breast Cancer Res Treat. 2014;145(3):775-84.

17. Rashid MU, Muhammad N, Naeemi H, Khan FA, Hassan M, Faisal S, et al. Spectrum and prevalence of BRCA1/2 germline mutations in Pakistani breast cancer patients: results from a large comprehensive study. Hered Cancer Clin Pract. 2019;17(1):27.

18. Rashid MU, Zaidi A, Torres D, Sultan F, Benner A, Naqvi B, et al. Prevalence of BRCA1 and BRCA2 mutations in Pakistani breast and ovarian cancer patients. Int J Cancer. 2006;119(12):2832-9.

19. Rashid MU, Muzaffar M, Khan FA, Kabisch M, Muhammad N, Faiz S, et al. Association between the Bsml polymorphism in the vitamin D receptor gene and Breast Cancer risk: results from a Pakistani case-control study. PLoS One. 2015;10(10):e0141562.

20. Rashid MU, Muhammad N, Bajwa S, Faisal S, Tahseen M, Bermejo JL, et al. High prevalence and predominance of BRCA1 germline mutations in Pakistani triple-negative breast cancer patients. BMC Cancer. 2016;16(1):673.

21. Nykamp K, Anderson M, Powers M, Garcia J, Herrera B, Ho YY, et al. Sherloc: a comprehensive refinement of the ACMG-AMP variant classification criteria. Genet Med. 2017:19(10):1105-17.

22. Cotton RT, Li D, Scherer SE, Muzny DM, Hodges SE, Catania RL, et al. Single nucleotide polymorphism in RECQL and survival in resectable pancreatic adenocarcinoma. HPB (Oxford). 2009;11(5):435-44.

23. Bowden AR, Tischkowitz M. Clinical implications of germline mutations in breast cancer genes: RECQL. Breast Cancer Res Treat. 2019;174(3):553-60.

24. Nguyen-Dumont T, Myszka A, Karpinski P, Sasiadek MM, Akopyan H, Hammet $F$, et al. FANCM and RECQL genetic variants and breast cancer susceptibility: relevance to South Poland and West Ukraine. BMC Med Genet. 2018;19(1):12

25. Pike AC, Shrestha B, Popuri V, Burgess-Brown N, Muzzolini L, Costantini S, et al. Structure of the human RECQ1 helicase reveals a putative strandseparation pin. Proc Natl Acad Sci U S A. 2009;106(4):1039-44.

\section{Publisher's Note}

Springer Nature remains neutral with regard to jurisdictional claims in published maps and institutional affiliations. 